\title{
Progressive Neurological Decline with Deep Bilateral Imaging Changes: A Protean Presentation of Dural Arteriovenous Fistulae
}

\author{
Rene A. Colorado ${ }^{a}$ Marcelo Matiello ${ }^{a}$ Hyun-Sik Yang ${ }^{a}$ \\ James D. Rabinov ${ }^{b}$ Aman Patel $^{b}$ Joshua A. Hirsch ${ }^{c}$ Ram Chavalid $^{d}$ \\ Thabele M. Leslie-Mazwi ${ }^{a, b}$ \\ a Department of Neurology, Brigham and Women's Hospital/Massachusetts General \\ Hospital/Harvard Medical School, Boston, MA, USA; ${ }^{b}$ Department of Neurosurgery, \\ Massachusetts General Hospital/Harvard Medical School, Boston, MA, USA; 'Department \\ of Radiology, Massachusetts General Hospital/Harvard Medical School, Boston, MA, USA; \\ ${ }^{\mathrm{d}}$ Departments of Neurosurgery and Radiology, Brigham and Women's Hospital/Harvard \\ Medical School, Boston, MA, USA
}

\section{Keywords}

Dural arteriovenous fistula $\cdot$ Neurological decline $\cdot$ Deep venous system · Venous hypertension $\cdot \mathrm{MRI} \cdot$ Bilateral edema Onyx embolization

\begin{abstract}
Intracranial dural arteriovenous fistulae (DAVF) within the deep cerebral vasculature are diagnostically challenging because of their variable clinical presentation and typical bilateral neuroimaging findings mimicking inflammatory, infectious, and metabolic processes. Increasingly, reports have emerged highlighting the diagnostic and treatment challenges of these lesions and their associated high morbidity and rapid clinical deterioration when untreated. We describe here a case series of 4 patients with deep cerebral DAVF who presented with impaired arousal or memory and behavioral changes. In all patients, the initial differential diagnosis included metabolic, inflammatory, infectious, or neoplastic disease, with an eventual correct diagnosis obtained after catheter angiography had demonstrated arterialization of the deep venous structures, including the vein of Galen. All patients were successfully treated with endovascular embolization, with 1 patient requiring additional surgical treatment. We review the contemporary diagnostic evaluation and management of DAVF within the deep cerebral vasculature. With rapid diagnosis and treatment, a favorable outcome is possible.
\end{abstract}




\section{Introduction}

Intracranial dural arteriovenous fistulae (DAVF) are a heterogeneous group of conditions involving pathological connections between arterial and venous components of the vasculature within the dural coverings of the brain. These lesions have variable clinical presentations and imaging findings. Intracranial DAVF can have unusual presentations and be particularly challenging to diagnose when they cause increasing venous congestion within deep central cerebral structures (e.g., basal ganglia, thalamus, and brainstem) and result in progressive neurological decline.

Furthermore, neuroimaging of these deep shunting lesions typically demonstrates bilateral findings that mimic other more common diseases including infiltrative neoplasms, inflammatory conditions, infectious encephalitides, and metabolic processes. Increasingly, deep cerebral DAVF are being recognized as a cause of these presentations and imaging findings. Case reports have emerged over the prior decade showing that this type of presentation has led to misdiagnoses that include Creutzfeldt-Jakob disease [1], progressive dementia [2, 3], parkinsonism [4], brainstem glioma [5-7], and viral encephalitis [6].

Given the high morbidity and potential rapid deterioration associated with arteriovenous shunting causing venous congestion within the deep cerebral vasculature and the remedial nature of these lesions, rapid diagnosis and therapy is imperative. We describe here an institutional review board-approved case series of this entity, derived over a 3-year period (June 2012 to July 2015), detailing the presentation, imaging, and successful treatment of 4 consecutive patients presenting with progressive neurological decline and associated bilateral imaging changes due to DAVF. We review the contemporary diagnostic approach and management of DAVF within the deep cerebral vasculature.

\section{Cases}

Table 1 summarizes the 4 patients and their clinical, imaging, and therapeutic characteristics. All presentations involved arousal or memory and behavioral changes. All patients demonstrated bilateral deep imaging changes on MRI (Fig. 1a) and arterialization of the deep venous structures, including the vein of Galen on angiography (Fig. 1b, 2). All were treated successfully with embolization as first-line therapy. Figure 1 emphasizes common MRI and angiography findings. We present 2 illustrative cases in greater detail.

Case 1

A 62-year-old woman presented with confusion, falls, and urinary incontinence progressing over 4 months. Parkinsonian features, including tremor, severe lead pipe rigidity, and bradykinesia with impaired balance were noted at her local hospital. A brain MRI showed bithalamic T2 hyperintensity with mild mass effect (Fig. 3a) and punctate susceptibility signal on gradient echo. Lumbar puncture (LP) was performed, with mildly sanguineous CSF. Her clinical status worsened immediately following the LP, prompting transfer to our institution. She was able to follow simple commands but was mute, hypertonic, and diffusely rigid. Head CT showed a new thalamic hemorrhage, with CT angiogram and venogram raising concern for a DAVF (Fig. 3b). Catheter angiography showed DAVF of the straight sinus with multiple feeding arteries (Fig. 3c, d). She was successfully treated with Onyx (Covidien, Dublin, Ireland) embolization with no residual early venous drainage (Fig. 3e). A thrombus was visualized in the vein of Galen and straight sinus on postoperative MRI. Anticoagulation was commenced to prevent rapid thrombosis with unwanted retrograde extension. A follow-up scan after 3 days showed interval improvement of thalamic edema, and the patient gradually recovered to become verbal again, with improvement in rigidity (without use of dopaminergic agents) in the 5 days following the procedure prior to her transfer to a rehabilitation unit. Follow-up 3 months later revealed clinical improvement with normal tone and no bradykinesia, though balance impairment still required use of a walker. There was corresponding resolution of bithalamic T2 hyperintensity on brain MRI (Fig. 3f) with no residual DAVF on catheter angiography. 
Colorado et al.: Progressive Neurological Decline with Deep Bilateral Imaging Changes: A Protean Presentation of Dural Arteriovenous Fistulae

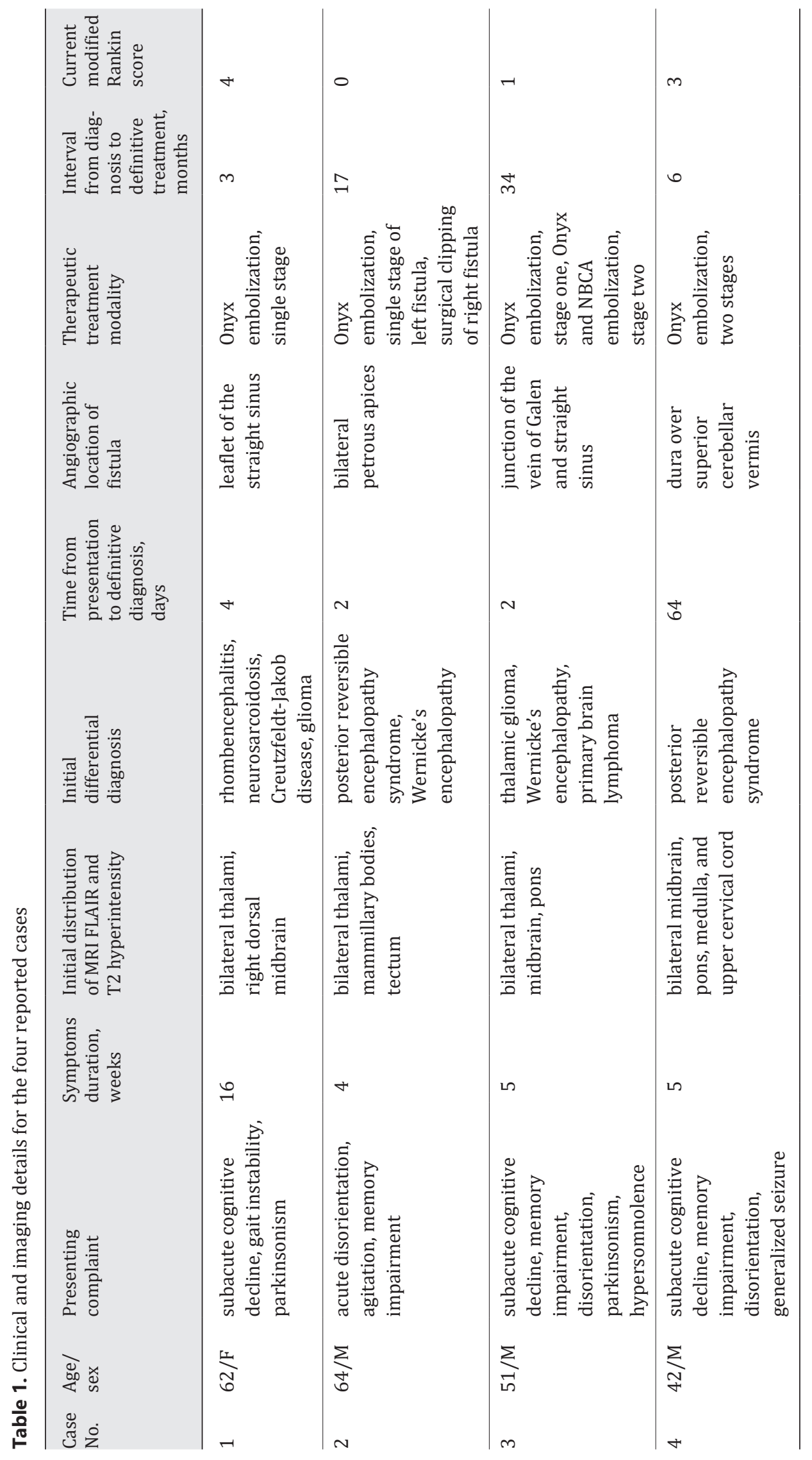




\section{Interventional Neurology}

\begin{tabular}{l|l}
\hline Intervent Neurol 2018;7:256-264 \\
\hline DOI: 10.1159/000487332 & $\begin{array}{l}\text { (c) 2018 S. Karger AG, Basel } \\
\text { www.karger.com/ine }\end{array}$ \\
\hline
\end{tabular}

Colorado et al.: Progressive Neurological Decline with Deep Bilateral Imaging Changes: A Protean Presentation of Dural Arteriovenous Fistulae

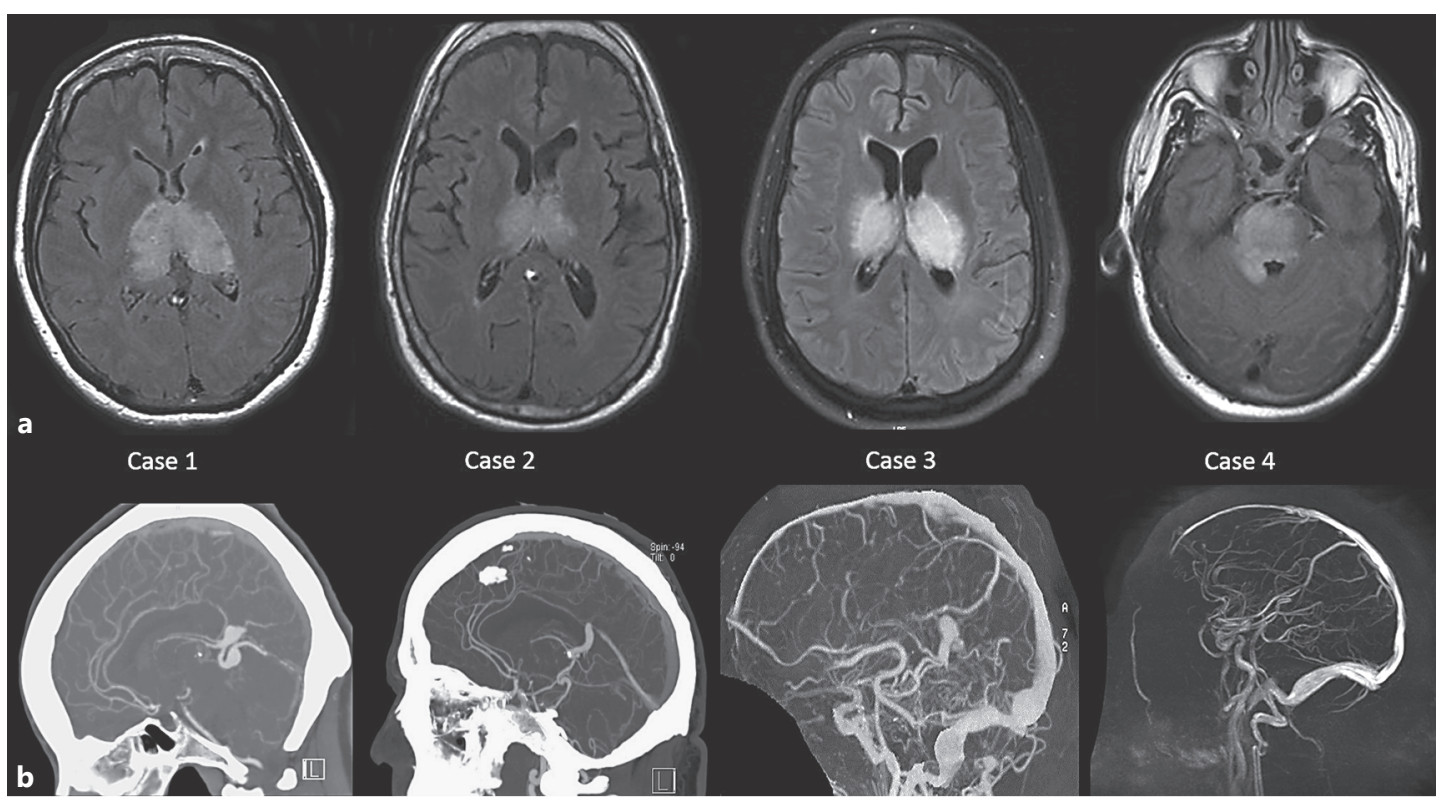

Fig. 1. a Axial FLAIR MRI images for each of the 4 patients demonstrating bilateral hyperintensity involving deep midline structures as a result of venous congestion. b Noninvasive vascular imaging: sagittal MIP reconstructions with CTA/CTV (cases 1 and 2) and MRA/MRV (cases 3 and 4) demonstrating abnormality of deep venous structures, either variceal (cases 1, 2, and 3) or thrombosed and absent (case 4). Note that the vein of Galen is abnormal in all cases.

Case 2

A 64-year-old man with hypertension and alcoholism presented with acute onset of confusion. On arrival, blood pressure was 236/108 $\mathrm{mm} \mathrm{Hg}$ and examination revealed disorientation, perseveration, confabulation, severe memory impairment, inattention, and limited speech. There were no focal sensorimotor deficits. Head CT was unrevealing and hypertensive encephalopathy was diagnosed. Subsequent MRI revealed bilateral T2 hyperintensities in the thalami, mammillary bodies, tectum, and periaqueductal grey matter (Fig. 4a). Thiamine was given for presumed Wernicke's encephalopathy. Electroencephalogram and CSF studies were normal. MRA showed poorly visualized dilated venous structures emptying into the vein of Galen, findings confirmed subsequently on CTA (Fig. 4b). Catheter angiography demonstrated a Cognard type IV DAVF in the left superior petrous ridge and a smaller Cognard type III DAVF in the right superior petrous ridge with shared drainage (Fig. 4c, d). The left lesion was treated successfully with Onyx embolization, with significant improvement (clinical and imaging) (Fig. 4e) over several days. The right lesion offered no pedicle to embolize from and the patient was treated 2 weeks later with microsurgical clipping (Fig. $4 \mathrm{f}$ ). Immediate postsurgical, 3-month, and 1-year follow-up catheter angiography showed no evidence of residual DAVF. He made a full neurological recovery.

\section{Discussion}

DAVF account for approximately $10-15 \%$ of all arteriovenous malformations [8]. The majority of these lesions are symptomatic due to the venous component, presenting in the most extreme forms with intracerebral hemorrhage. With deep DAVF, clinical presentation is highly variable, with venous congestion prominently involving the midbrain, pons, thalami, and basal ganglia. As a result, cognitive impairment, memory impairment, inattention, gait abnormalities, parkinsonism, personality changes, and hypersomnolence are prominent symptoms, as highlighted in our case series. Progression of symptoms as seen in our series 

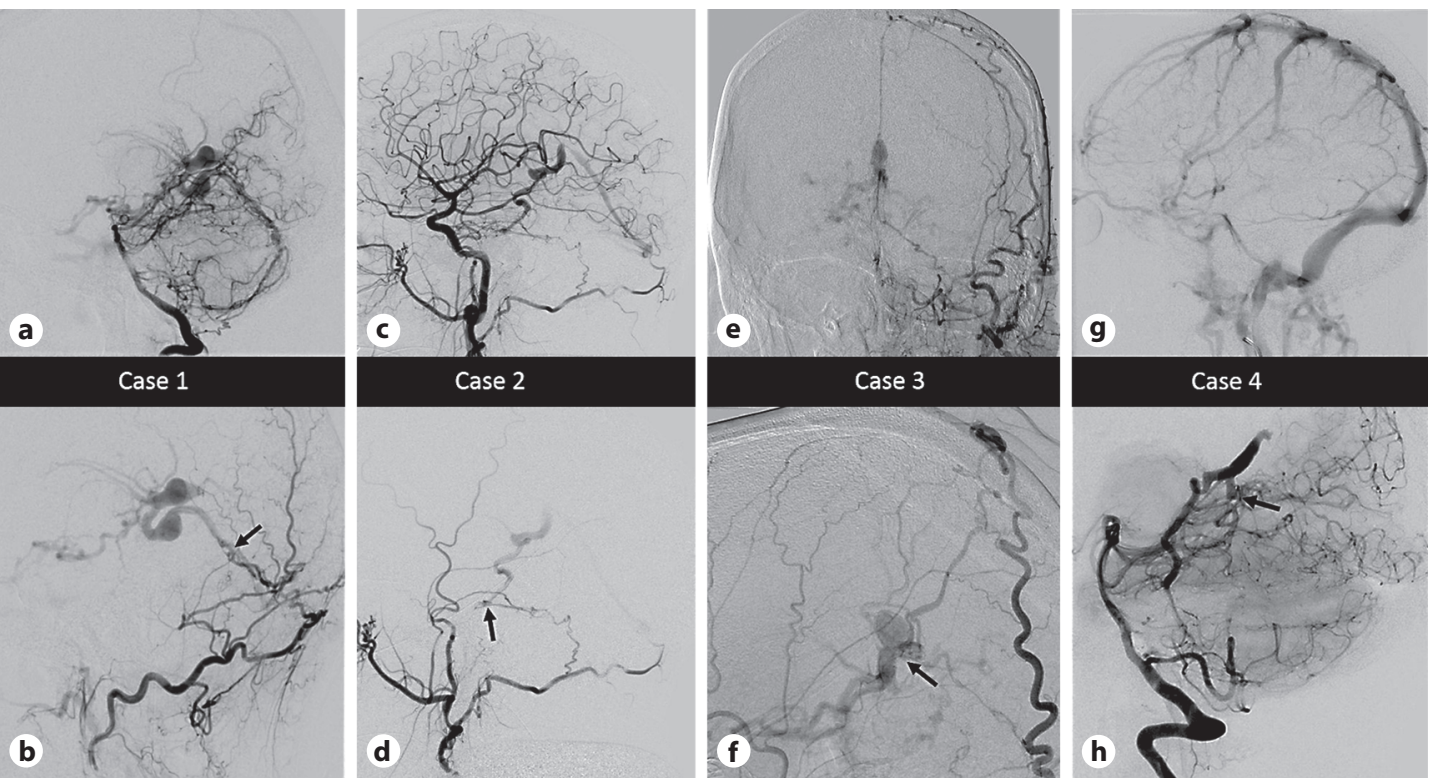

b

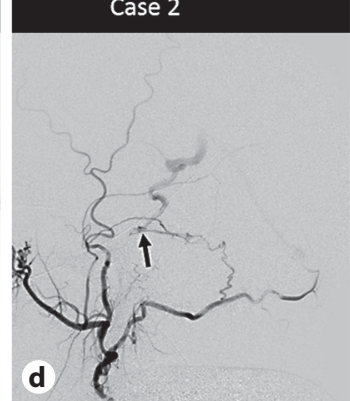

Colorado et al.: Progressive Neurological Decline with Deep Bilateral Imaging Changes: A Protean Presentation of Dural Arteriovenous Fistulae

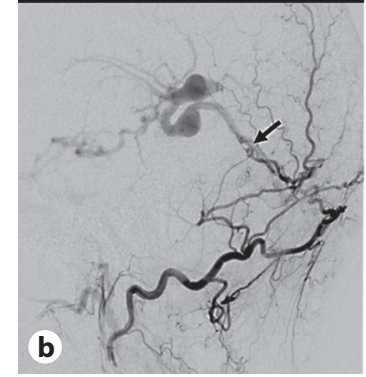

Fig. 2. Select angiographic images for the patients described. In the lower row, the black arrows point to the location of the fistulous connection. In all cases multiple arterial feeders from both dural and parenchymal vessels were present, and select images are shown to best illustrate anatomical considerations. a, b Case 1. a Lateral angiogram, right vertebral artery injection, mid-arterial phase demonstrating enlargement of the posterior meningeal artery and artery of the falx cerebelli feeding a dural fistula with drainage into variceal superior hemispheric veins and the vein of Galen. b Lateral magnified angiogram, left occipital artery injection, mid-arterial phase demonstrating transosseous supply and venous fistula anatomy in greater detail. c, d Case 2. c Lateral angiogram, right common carotid injection, mid-arterial phase showing right meningohypophyseal trunk and right occipital artery supply to a point of fistulation along the right petrous apex. Variceal venous structures are then seen to fill in the arterial phase, including faint opacification of the straight sinus. $\mathbf{d}$ Lateral angiogram, right external carotid injection, mid-arterial phase showing transosseous dural supply from the occipital artery isolating the point of fistulation. e, $\mathbf{f}$ Case 3. e Anteroposterior angiogram, left external carotid injection, late arterial phase showing right transosseous superficial temporal artery, middle meningeal artery, and occipital artery feeders sending dural falcine branches deep to the point of fistulation adjacent to the vein of Galen, which is variceal and seen to fill in the arterial phase. $\mathbf{f}$ Lateral magnified view, right external carotid injection, late arterial phase showing similar anatomy on the contralateral side with large dural falcine branches and point of fistulation on the lateral wall of the vein of Galen. g, $\mathbf{h}$ Case 4. $\mathbf{g}$ Lateral angiogram, left internal carotid artery injection, venous phase showing absence of deep venous structures. The structures did not opacify in the venous phase on any arterial injections, due to a combination of arterialized flow and thrombosis. $\mathbf{h}$ Lateral magnified angiogram, right vertebral injection demonstrating a midline fistula fed by bilateral superior cerebellar arteries with drainage into a dilated basal vein of Rosenthal and retrograde into lateral pontomesencephalic veins. The vein of Galen was thrombosed and did not opacify.

and in prior case reports [1-7] typically ranges from months to days, rarely years [4]. The clinical course therefore does not offer a diagnostic clue to a vascular etiology. Progression of venous hypertension in untreated DAVF eventually leads to venous infarction, hemorrhage, or more severe neurological decline including coma.

Diagnostic evaluation aims to identify the location of the shunt and impact on brain parenchyma and to define arterial supply and pattern of venous drainage. Deep diffuse or bilateral changes on MRI or CT should prompt vascular imaging with MRA/CTA (including venography) for all patients, and any abnormality of vasculature, particularly abnormally enlarged or tortuous vessels or abnormal venous sinuses, should prompt consideration of 


\section{Interventional Neurology}
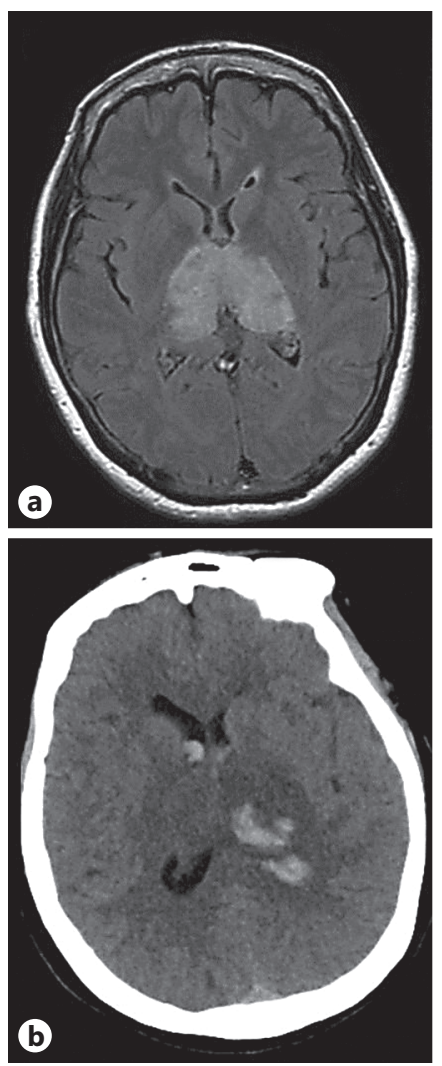

Intervent Neurol 2018;7:256-264

DOI: $10.1159 / 000487332$

(c) 2018 S. Karger AG, Basel
www.karger.com/ine

Colorado et al.: Progressive Neurological Decline with Deep Bilateral Imaging Changes: A Protean Presentation of Dural Arteriovenous Fistulae
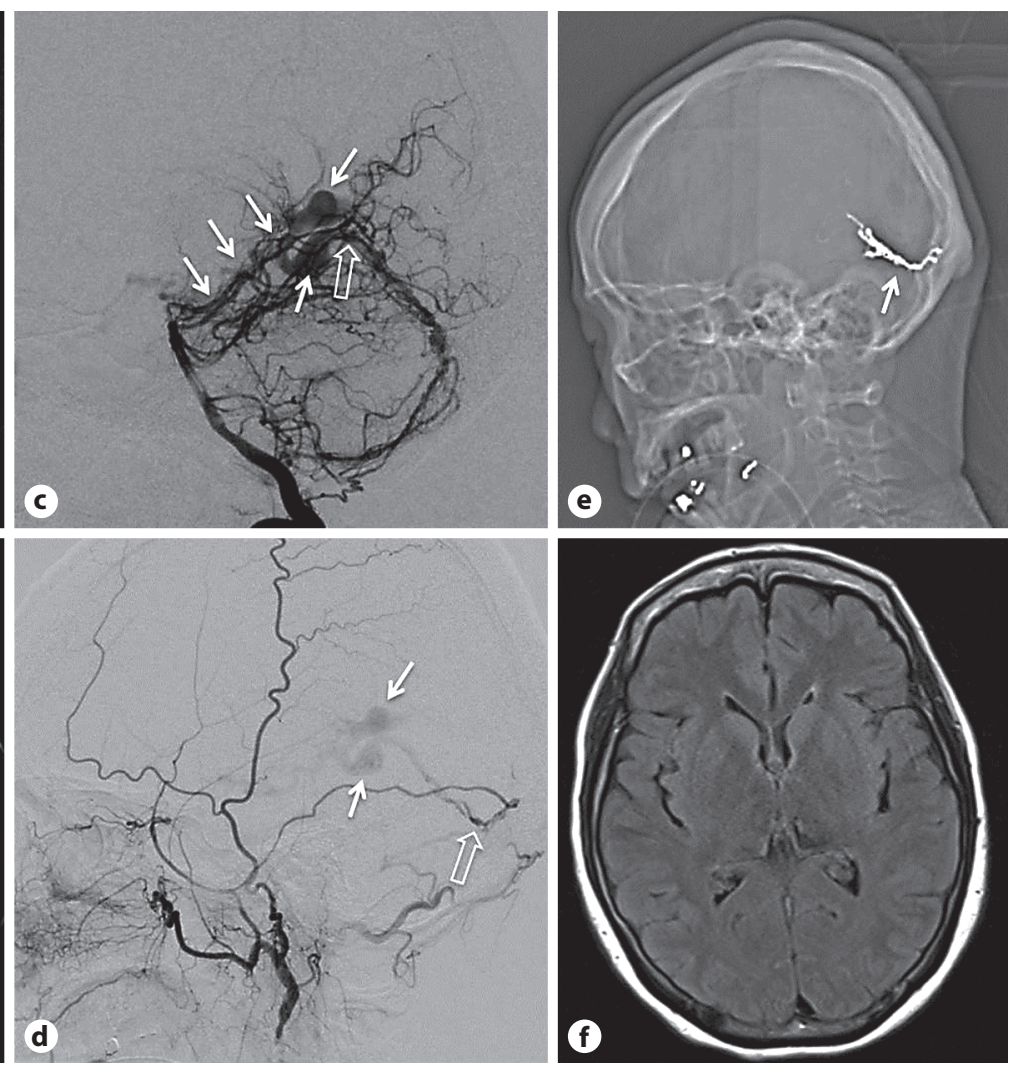

Fig. 3. a Initial axial FLAIR MRI showing bilateral thalamic hyperintensity. b Axial CT showing hemorrhagic change within the congested tissue of the left thalamus with intraventricular extension. c Digital subtraction angiography right vertebral injection, lateral view showing enlargement of the posterior meningeal artery and artery of the falx cerebelli feeding a dural fistula (open arrow) with drainage into variceal deep venous structures (white arrows) that fill in the arterial phase. d Digital subtraction angiography, left external carotid injection, lateral view showing left middle meningeal artery feeder to the same point of fistulation (open arrow), with similar venous egress (white arrows). e Scout image from post-procedural CT showing Onyx cast following deposition in the middle meningeal supply successfully occluding the point of fistulation (white arrow). f FLAIR MRI 3 months post-procedure showing slowly improving deep tissue changes.

arteriovenous shunt. Suspicion for an arteriovenous shunt should increase if infarction or hemorrhage is seen in an unusual location or age group [9]. Even in the absence of vessel abnormalities on noninvasive imaging, a high level of suspicion should be maintained in the appropriate clinical context, as noninvasive imaging may have low diagnostic accuracy of DAVF within deep cerebral veins [10]. Cervical cord MRI/MRA should be considered in suspected deep DAVF, particularly when involving the lower brainstem. Dynamic studies with MRA or CTA may provide further details.

Digital subtraction angiography (DSA) remains the gold standard and is required to fully define the lesion and stratify risk. DSA should be considered for all patients for whom vascular etiology is suggested by noninvasive studies. For complete evaluation, a six-vessel DSA with bilateral selective catheterization of the external and internal carotid arteries and both vertebral arteries is required for clear identification of the therapeutic target. In select cases, cervical vessels should be imaged as DAVF within the high cervical cord may result in brainstem signal changes [5]. 


\section{Interventional Neurology}
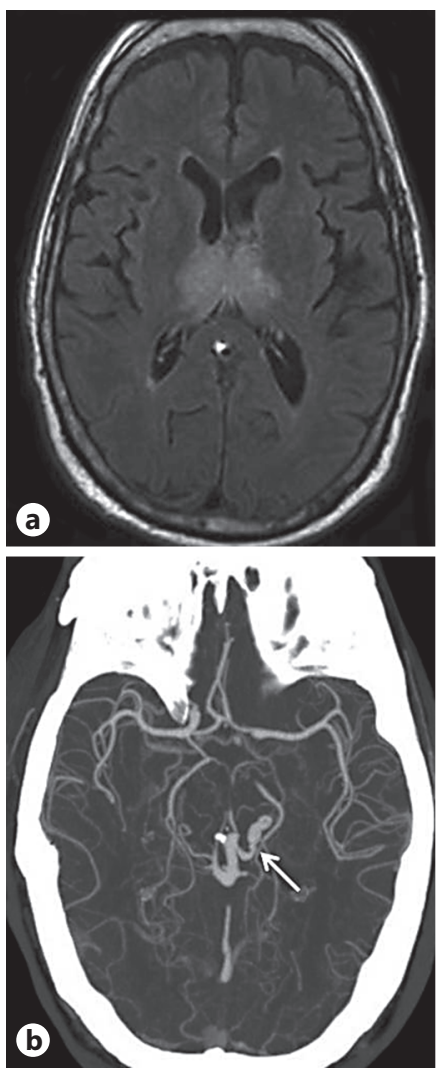

Colorado et al.: Progressive Neurological Decline with Deep Bilateral Imaging Changes: A Protean Presentation of Dural Arteriovenous Fistulae
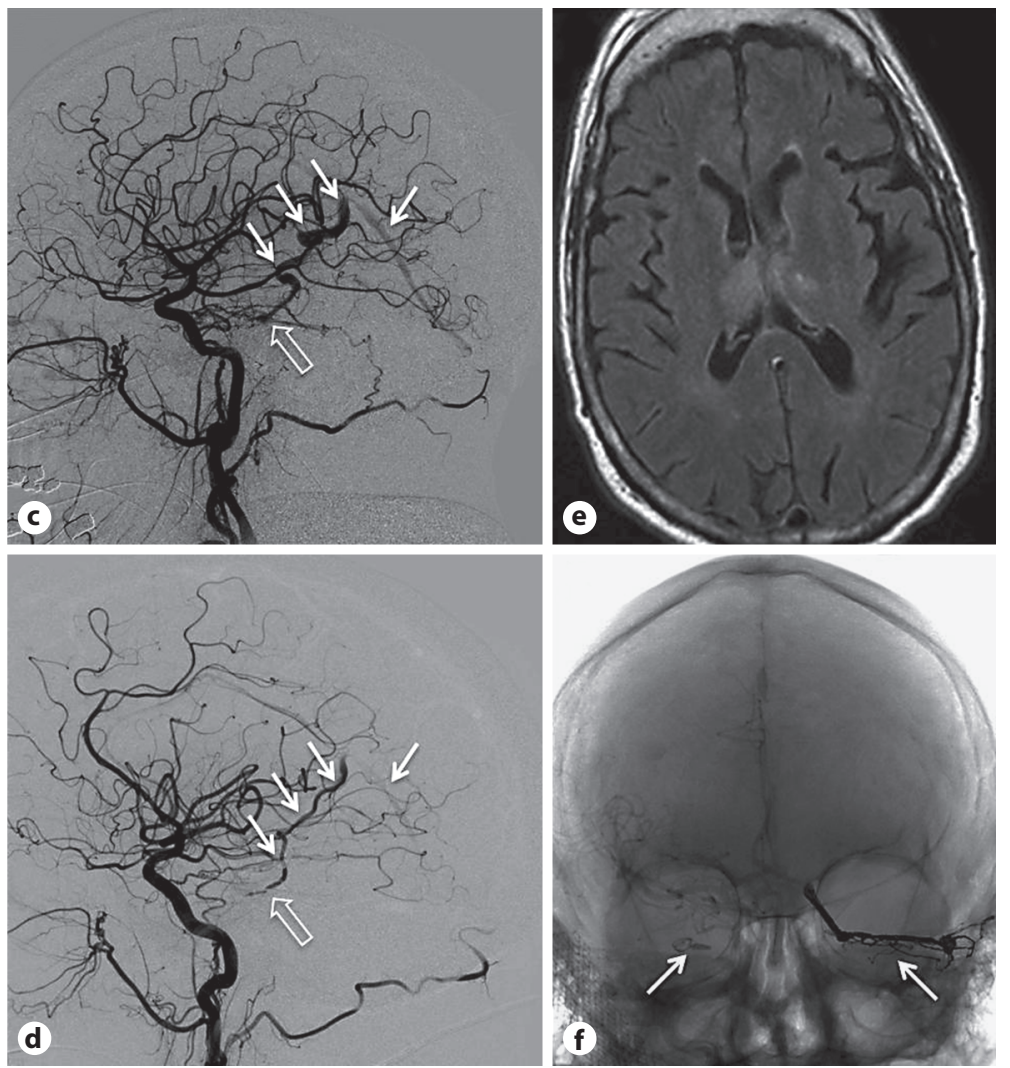

Fig. 4. a Initial FLAIR MRI showing bilateral thalamic hyperintensity. $\mathbf{b}$ CTA axial MIP demonstrating variceal deep venous structures (white arrow) concerning an arteriovenous shunt. c Digital subtraction angiography, right common carotid injection, lateral magnified view showing right meningohypophyseal trunk supply to a point of fistulation along the right petrous apex (open arrow); variceal venous structures (white arrows) are then seen to fill in the arterial phase, including faint opacification of the straight sinus. $\mathbf{d}$ Digital subtraction angiography, left common carotid injection, lateral magnified view showing left meningohypophyseal trunk supply to a second and independent point of fistulation along the left petrous apex (open arrow) with similar venous egress (white arrows). e Axial FLAIR MRI 5 days after initial embolization showing early improvement of signal changes. $\mathbf{f}$ Unsubtracted anteroposterior angiography showing an Onyx cast occluding the left fistulous pedicle and a clip occluding the right fistulous pedicle by occlusion of the proximal venous egress (white arrows).

LP is sometimes undertaken during initial workup of an undiagnosed DAVF. Rapid severe neurological decline, as in one of our patients, has been described after LP in patients with DAVF, likely related to an acute differential pressure created by the sudden decrease in intrathecal and intracranial pressure after CSF diversion $[11,12]$. LP or CSF diversion should be avoided in any patient suspected to have a DAVF, and in any patient with a suspected vascular cause and with acute neurological deterioration after LP for a suspected nonvascular process.

DAVF presenting with bilateral deep signal changes and progressive neurological decline will invariably require invasive treatment, given the high morbidity and mortality associated with these lesions. In our opinion, treatment should be performed expeditiously with the goal of venous decompression by obliteration of the point of connection between the arterial and venous components of the lesion. Lesions presenting with hemorrhage, venous infarction, diffuse bilateral edema, and rapidly declining symptoms warrant more emergent treatment $(<4 \mathrm{~h})$. 
Outcome is dependent on the time to diagnosis as well as the treatment and degree of chronic edema or vascular complications (infarction or hemorrhage). Spontaneous regression of DAVF in general is low [13]. However, if treatment is timely and successful, complete recovery is possible. Resolution of MRI findings typically lags behind clinical recovery, and in occasional cases imaging changes may be permanent.

Endovascular treatment with a transarterial approach is typically the preferred method for deep DAVF. The transvenous approach occasionally utilized to treat intracranial DAVF is challenging in cases involving the deep venous system because of the tortuosity, caliber, and fragility of the venous structures in this region. To gain definitive cure, the therapeutic target is complete occlusion of both the fistula itself and the proximal venous segment of the DAVF.

Most DAVF can be cured by endovascular treatment alone, but multiple staged treatments may be required with complex DAVF or if a high load of radiation or contrast is expected. In staged cases, the initial target should be the vessel that would produce the greatest decrease in the venous pressure gradient. If obliteration of the DAVF is not possible with endovascular interventions, surgical approaches can provide treatment options either alone or in combination with endovascular treatment. A less desirable approach is radiation therapy because of the delay to occlusion and low cure rates when uses as primary treatment [14].

The follow-up of these patients may be divided into two phases: staged and post-cure. If a staged treatment approach is necessary, we advocate for brief intervals between treatments (we aim for ideally less than 2 weeks) based on patient morbidity from the natural history of the condition. We did not use corticosteroids or hypertonic treatment in any case because of uncertainty regarding the benefit of these agents for treatment of venous congestion and edema in this condition. In the absence of data to guide blood pressure goals after a diagnosis has been made, it is reasonable to aim for normotension, though procedurally mild hypotension was induced.

Therapeutic anticoagulation can be considered in patients with evidence of venous thrombosis if acute sinus thrombosis is felt to be the driving factor of acute decline (e.g., acutely occluded straight sinus), but it should be carefully considered given the risk of hemorrhage in these patients. Importantly, therapeutic anticoagulation should not be thought of as a therapy that is curative or extends the window to definitive treatment with invasive approaches. Only one of our cases was treated with post-procedure anticoagulation to prevent exuberant thrombosis in the variceal venous components of the treated fistula. Mechanical recanalization of venous sinus thrombosis may be considered if that recanalization will provide an egress route for pressurized flow, but deep veins are not typically suitable endovascular targets. If pursued, sinus recanalization maneuvers are not curative, but merely helpful for decreasing venous congestion. We avoid these approaches as a general rule.

After the DAVF has been completely occluded, noninvasive follow-up with MRI should be undertaken to follow expected tissue changes, with timing dependent on the rate of clinical improvement of the patient. We follow up with MRI at intervals of at least 1 month, 3 months, and 1 year post-treatment in patients improving as anticipated. We also perform a follow-up DSA at least at 3 months and 1 year post-treatment after definitive occlusion. If concern for persistent fistula exists, we repeat DSA at 1 month in addition to the above time points. This imaging approach is based on our historical approach to DAVF patients [15]. Given the prominent cognitive symptoms seen in these patients, we pursue formal neuropsychiatric testing and aggressive cognitive rehabilitation to optimize outcomes in this unusual population.

This series is subject to the usual limitations of a small case series. However, for rare pathologies, case series represent an important means of presenting unusual clinical information. Further, we can only estimate a denominator for the total number of DAVF cases evaluated and therefore cannot provide an accurate local incidence of this entity. Conversely, potential cases may have been missed diagnostically, further confounding incidence calculations for this infrequent condition. 


\section{Conclusions}

Deep venous congestion has a protean appearance on MRI and should raise concern for a deep vascular shunt, most typically a DAVF. Clinical suspicion must be high to make a timely diagnosis. With rapid diagnosis and treatment, a favorable outcome is possible for these patients.

\section{Statement of Ethics}

The subjects gave informed consent and the study protocol was approved by the institute's committee on human research.

\section{Disclosure Statement}

The authors declare that there is no conflict of interest. This research received no specific grant from any funding agency in the public, commercial, or not-for-profit sectors.

\section{References}

1 Chahbazian K, Théaudin M, Lehmann P, Sachet M, Adams D, Saliou G: Reversible pseudo-Creutzfeldt-Jakob syndrome related to cerebral dural arteriovenous fistula. J Am Geriatr Soc 2014;62:2024-2026.

2 Tanaka K, Morooka Y, Nakagawa Y, Shimizu S: Dural arteriovenous malformation manifesting as dementia due to ischaemia in bilateral thalami. Surg Neurol 1999;51:489-493.

3 Holekamp TF, Mollman ME, Murphy RK, Kolar GR, Kramer NM, Derdeyn CP, Moran CJ, Perrin RJ, Rich KM, Lanzino G, Zipfel GJ: Dural arteriovenous fistula-induced thalamic dementia: report of 4 cases. J Neurosurg 2016;124:1752-1765.

4 Luo Y, Qi J, Cen Z, Hu H, Jiang B, Luo W: Two cases of dural arteriovenous fistula presenting with parkinsonism and progressive cognitive dysfunction. J Neurol Sci 2014;343:211-214.

5 Crum BA, Link M: Intracranial dural arteriovenous fistula mimicking brainstem neoplasm. Neurology 2004; 62:2330-2331.

6 Borja MJ, Schaefer PW, Boulter DJ: Case of the season: dural arteriovenous fistula mimicking a bithalamic neoplasm or viral encephalitis. Semin Roentgenol 2014;49:4-9.

7 Le Guennec L, Leclercq D, Szatmary Z, Idbaih A, Reyes-Botero G, Delattre JY, Psimaras D: Dural arteriovenous fistula mimicking a brainstem glioma. J Neuroimaging 2015;25:1053-1055.

8 Newton TH, Cronqvist S: Involvement of dural arteries in intracranial arteriovenous malformations. Radiology 1969;93:1071-1078.

9 Geibprasert S, Pongpech S, Jiarakongmun P, Shroff MM, Armstrong DC, Krings T: Radiologic assessment of brain arteriovenous malformations: what clinicians need to know. Radiographics 2010;30:483-501.

10 Kitajima M, Hirai T, Korogi Y, Yamura M, Kawanaka K, Ikushima I, Hayashida Y, Yamashita Y, Kuratsu J: Retrograde cortical and deep venous drainage in patients with intracranial dural arteriovenous fistulas: comparison of MR imaging and angiographic findings. AJNR Am J Neuroradiol 2005;26:1532-1538.

11 Cognard C, Gobin YP, Pierot L, Bailly AL, Houdart E, Casasco A, Chiras J, Merland JJ: Cerebral dural arteriovenous fistulas: clinical and angiographic correlation with a revised classification of venous drainage. Radiology 1995;194:671-680.

12 Rastogi S, Liebeskind DS, Zager EL, Volpe NJ, Weigele JB, Hurst RW: Rapid cognitive decline following lumbar puncture in a patient with a dural arteriovenous fistula. Surg Neurol 2004;62:341-345.

13 Luciani A, Houdart E, Mounayer C, Saint Maurice JP, Merland JJ: Spontaneous closure of dural arteriovenous fistulas: report of three cases and review of the literature. AJNR Am J Neuroradiol 2001;22:992-996.

14 Gandhi D, Chen J, Pearl M, Huang J, Gemmete JJ, Kathuria S: Intracranial dural arteriovenous fistulas: classification, imaging findings, and treatment. AJNR Am J Neuroradiol 2012;33:1007-1013.

15 Chandra RV, Leslie-Mazwi TM, Mehta BP, Yoo AJ, Rabinov JD, Pryor JC, Hirsch JA, Nogueira RG: Transarterial Onyx embolization of cranial dural arteriovenous fistulas: long-term follow-up. AJNR Am J Neuroradiol 2014; 35:1793-1797. 